\title{
La caracterización de las políticas ambientales de la Unión Europea
}

\section{Carmen Navarro Gómez *}

1. La ubicación de los problemas ambientales en la agenda de la Unión Europea

La acción pública se construye para dar solución a un problema o satisfacer una carencia en la sociedad. En concreto, en nuestras sociedades occidentales, en las que se han generado nuevos espacios de formulación de demandas sociales, el traslado de parte de ellas a la esfera política ha provocado una expansión sin-precedentes del alcance de la agenda de actuación pública (incluida la agenda comunitaria), así como un crecimiento de los recursos financieros para desarrollar las acciones públicas '. Entre ellas, las políticas para la protección del medio ambiente, se situarían en un lugar privilegiado de dicha agenda.

Y si bien es cierto que en la teoría del análisis de las decisiones públicas se mantiene que no existen problemas objetivos, sino percepciones subjetivas que califican un determinado fenómeno como problema social, lo cierto es que en la esfera de las cuestiones relacionadas con el medio ambiente, el problema que representa su agudo deterioro no admite discusión en cuanto a su existencia y la necesidad de una intervención activa por parte de los poderes públicos para paliarlo. No obstante, y pese a tener una base real, también está afectado de un cierto componente de construcción subjetiva, puesto que:
- no existe una percepción unánime sobre la gravedad y las características del problema, piénsese, por ejemplo, en la diferente forma de percibir un problema ambiental por parte de las sociedades nórdicas y de las sociedades latinas ${ }^{2}$;

- el problema del deterioro del medio ambiente no es independiente de otros problemas y, además, suele construirse en conflicto con ellos, de modo que nace en relación dialéctica con otras carencias, lo que le priva de autonomía en su consideración. Junto a la prioridad de la protección del medio ambiente, existen otras (desarrollo industrial, producción energética, empleo, producción agrícola, etc.) que pueden dificultar la correcta ubicación de los problemas ambientales en la agenda pública y generar un ambiente de conflicto:

- se tiende a vincular el problema con la solución del mismo, de modo que la manera de plantearlo suele ser instrumental, construida desde una perspectiva que permita una acción con resultados satisfactorios. Esta circunstancia -común, por otra parte, a gran parte de las formulaciones de las decisiones públicas - se compadece mal con el carácter singular de los problemas ambientales, en torno a los cuales existe un alto grado de incertidumbre sobre el impacto o las repercusiones futuras de una acción o falta de acción, debido a las carencias en el conocimiento de las reglas de funcionamiento del medio ambiente (la interacción entre los sistemas naturales).

Con independencia de los rasgos concretos que caractericen la construcción de un problema ambiental, lo cierto es que una vez son identificados como problemas de relevancia pública, 
éstos pueden dar lugar o no a la acción de los poderes públicos. En un entorno de gobierno multinivel como es el que nos encontramos en el caso de los problemas ambientales, el centro del análisis está en determinar por qué el problema se sitúa en la agenda del "Gobierno Europeo" y no en la de otro.

Desde una perspectiva histórica, en la acción de la Comunidad Europea para la protección del medio ambiente, las causas de la incorporación inicial de los problemas ambientales en la agenda del Consejo estaban estrechamente ligadas a la consecución del mercado común y se explicitaban en:

- la aparición de obstáculos al comercio en relación a los productos. En efecto, la existencia de regulaciones nacionales ambientales diferentes podía resultar un obstáculo para el libre comercio por obligar a reducir los movimientos a productores que, al intentar introducirse en mercados de otros Estados miembros, se enfrentaban barreras técnicas o normativas que, si se querían superar, obligaban a repercutir tales cargas en los precios, lo cual creaba de facto, un obstáculo a la libre circulación;

- los diferentes tipos de controles ambientales que aplicaban las políticas nacionales a los procesos industriales, pues si bien es cierto que los requisitos de producción industrial no amenazan el libre comercio tan directamente como lo puede hacer la regulación de los productos, sin embargo, la adopción en uno o varios Estados de unos controles más estrictos que en otros incrementa los costes de producción de los fabricantes ubicados en ese territorio creando una ventaja competitiva para los productores de otros Estados.

La respuesta a estos problemas era la armonización de las legislaciones. Posteriormente, en los años ochenta y principios de los noventa, la política ambiental comunitaria deja de estar exclusivamente fundada en el "problema" de la consecución del mercado común y pasa a basarse en la consecución de valores ambientales en sí mismos. A finales de la década alcanza su máximo apogeo, hasta el punto de que la $\mathrm{CE}$ se convierte en un actor central en la conformación de las políticas nacionales dirigidas a la protección del medio ambiente. Según algunos autores ${ }^{3}$, en estos años, el proceso político en la arena ambiental está conducido por un pequeño número de Estados que poseen una políticas ambientales internas significativamente más progresistas que las de los demás (Alemania, Dinamarca y Holanda) que presionan por incluir los problemas en la agenda comunitaria a fin de proyectar sus políticas sobre el resto de los Estados miembros a través de la acción de la Comunidad. Según esta tesis, las políticas internas de los Estados más avanzados empujan. el proceso en Bruselas. Las decisiones, por tanto, se formulan por medio de un proceso de presiones para la eulropeización de las medidas nacionales de estos países líderes.
Se da en estas circunstancias la paradoja de que los problemas que estas acciones tienden a corregir son, en muchas ocasiones, específicos de los Estados promotores (lluvia ácida, contaminación de las aguas de los grandes ríos transnacionales que los atraviesan), y para los que el resto de los países tienen una menor sensibilidad y sin embargo logran situarse en la agenda comunitaria exigiendo una respuesta común ".

Pero, pese a que se reconozca este origen en algunas de las politicas ambientales comunitarias, no se puede generalizar para todas. No existen reglas generales sobre la construcción de los problemas ambientales en la Comunidad Europea. La acción ambiental está dividida en sectores y la lógica de la colocación de problemas en la agenda comunitaria varía enormemente de un sector a otro ${ }^{5}$. Con independencia de cuál haya sido la causa y las condiciones de la incorporación de un problema ambiental en la agenda de la Comunidad, lo cierto es que desde mediados de la década de los ochenta, asistimos a la emergencia de una agenda política europea: Europa se convierte cada día más en el lugar de debate, donde se definen los problemas, se califican y se barajan las alternativas para darles solución y donde los diferentes actores entran en conflicto o negociación. De ahí que se llegue a afirmar por algunos autores ${ }^{6}$ que, con la excepción de los mencionados países líderes ${ }^{7}$, el resto de los Estados miembros ha construido sus políticas ambientales casi exclusivamente en razón de la necesidad de adaptar las directivas comunitarias o de aplicar sus reglamentos. Hasta tal punto es determinante la existencia de normas europeas, que se mantiene que, en ausencia de las mismas, no existiría regulación ambiental sobre muchos sectores, de modo que para estos Estados, entre los que claramente se encuentra España, sus políticas ambientales $-\mathrm{y}$, por ende, sus legislaciones ambientales puesto que la mayor parte de las acciones comunitarias son políticas regulativas- están europeizadas.

Igualmente contribuye a facilitar la comprensión sobre la lógica de la colocación en la agenda europea de los problemas ambientales acercarse al ya clásico cuadro de WIISON ${ }^{8}$ adaptado (por Majone"), referente a la ubicación de los temas en uno u otro nivel de gobierno en función de las características de los problemas que estén en juego y de los costes que implique la solución de los mismos, de modo que:

\begin{tabular}{|c|c|c|c|}
\hline \multicolumn{4}{|c|}{ Beneficios } \\
\hline \multirow{3}{*}{ Costes } & & Difusos & Concentrados \\
\hline & Difusos & $\begin{array}{l}\text { politicas mayoritarias } \\
\text { (1) }\end{array}$ & $\begin{array}{l}\text { politicas clientelares } \\
\text { (3) }\end{array}$ \\
\hline & Concentrados & $\begin{array}{l}\text { politicas de } \\
\text { emprendedor (4) }\end{array}$ & $\begin{array}{l}\text { políicas de grupos } \\
\text { de interés (2) }\end{array}$ \\
\hline
\end{tabular}

Finente. WILSON, 1980 The politics of policy. 
Cuando los costes y los beneficios están muy distribuidos (1) los grupos de interés tienen poco incentivo en activarse alrededor de estos temas, porque ningún sector de la sociedad puede esperar beneficiarse de una parte desproporcionada de los beneficios o evitar una parte desproporcionada de los gastos. Por ello, estos temas se suelen tratar en la arena tradicional de las políticas mayoritarias (majoritarian politics). En el contexto europeo ello significa que este tipo de asuntos tienden a tratarse más en la arena nacional que en la supranacional, lo que explicaría, por ejemplo, que la mayor parte de las políticas ambientales que implican la construcción de infraestructuras permanece bajo un control estatal o subestatal.

En el cuadrante opuesto (2), cuando tanto los costes como los beneficios están concentrados, cada grupo tiene un fuerte incentivo para ejercer influencia política. Este tipo de políticas ambientales no está excluido de la agenda europea, siendo quizás la desarrollada a través del Fondo de Cohesión la que más se ajustaría a estos términos, en donde la dinámica de los grupos de interés estaría representada por las negociaciones intergubernamentales entre dos o más grupos de países.

Cuando los beneficios de una política ambiental están concentrados mientras que los costes están ampliamente distribuidos, los pequeños grupos organizados (empresas oligopólicas del automóvil, las industrias químicas o farmacéuticas, etc.) tienen grandes incentivos para ejercer presión a fin de obtener legislación favorable. Al tiempo, los consumidores tienen poco incentivo para organizarse dado que los costes de regulación son bajos en términos per capita. Se produce normalmente una situación de políticas clientelares con la posibilidad de que los decisores pueden ser capturados por los intereses organizados.

Finalmente, cuando una política puede conferir beneficios generales a un coste financiado principalmente por los agentes económicos causantes de la contaminación (4) (ej.: los costes de un aire y unas aguas más limpios, productos más seguros o menos ruidosos), el incentivo para organizarse es mayor para los oponentes de la política que para sus beneficiarios. En estas circunstancias, las medidas sólo se adoptarán si hay un empresario político que active la sensibilidad pública asociando la legislación a valores ampliamente compartidos (medio ambiente sano, salud y seguridad, etc.) y sitúe a los que soportarán los costes de la regulación en una situación defensiva que les impida oponerse a unas nuevas medidas más estrictas para el desarrollo de su actividad, pero intenten que el esfuerzo sea el menor posible. En el caso europeo, los problemas ambientales que se construyen con estas características tienen muchas posibilidades de situarse con éxito en la agenda europea debido a varios factores, entre otros, al aislamiento de la Comisión de las constricciones políticas nacionales y de los resultados electorales, al activismo del Tribunal de Justicia y al interés del Parlamento en buscar su propio papel. Ésos son los tres factores que explicarían por qué los intereses difusos suelen estar mejor representados en el nivel europeo que en el nivel nacional y por qué el papel promotor que a menudo desempeña la Comisión constituye un elemento determinante que, en muchos casos, contribuye a que los temas ambientales, en especial los que se refieren a la consecución de objetivos de calidad del medio, se sitúen en la agenda del gobierno europeo. Hay que constatar al respecto la existencia de una paradoja importante: las mismas instituciones supranacionales que son tan criticadas por su •déficit democrático* o por su distancia con respecto a los ciudadanos, pueden ser de hecho las mejores abogados de los intereses difusos que no encuentran una expresión adecuada en los sistemas políticos nacionales ${ }^{10}$.

\section{Los actores presentes a lo largo del proceso: actores públicos y privados, su peso y sus interacciones}

Hay que dejar claro de antemano que no se puede generalizar un conjunto de reglas aplicables a todos los sectores que conforman el ámbito ambiental y a todas las épocas del desarrollo de la construcción comunitaria. Las instituciones de la Comunidad interactúan entre ellas en modo tal que el contenido final de las medidas no puede ser previsto ni por los Estados que han promovido la medida, en su caso, ni por la Comisión si es que ésta ha actuado como promotora. Sin embargo, a lo largo de la dinámica que caracteriza este proceso se pueden detectar ciertos rasgos comunes al ámbito.

\subsection{La Comisión}

La Comisión es un actor esencial en el proceso de toma de decisiones de la Comunidad Europea. Esta institución ostenta formalmente el monopolio de la iniciativa de proceso de toma de decisiones y sigue estando presente a lo largo de todo su desarrollo, teniendo capacidad, gracias a la disposición del artículo 189 del Tratado de la Comunidad Europea (actual artículo 249 TCE) de influir hasta el último momento en la decisión.

Pero más que por su capacidad de influir en el procedimiento final de adopción de decisión de una medida, la clave del papel de esta institución se sitúa en las fases iniciales de 
gestación de una propuesta, debido fundamentalmente a que es ella la que en muchos casos actúa como policy entrepreneur de la propuesta, incorporando por sí misma el asunto en la agenda comunitaria y, en todo caso, introduciendo el enfoque propio que quiera dar a la propuesta de decisión, tras haber realizado, en la mayoría de las ocasiones, una amplia labor de consultas a los sectores implicados 0 , incluso, sometiendo la cuestión a la práctica del debate abierto sobre un asunto para todos los sectores implicados, a través de la fórmula de los Libros Verdes o los Libros Blancos.

\subsection{Parlamento Europeo}

No ha sido hasta la fecha un actor clave en el proceso de toma de decisiones, si bien ha venido desarrollando una labor permanente en pos de una mayor intervención de la Comunidad para la protección del medio ambiente, en especial a través de su Comisión de Medio Ambiente ${ }^{11}$, y también por medio de las resoluciones adoptadas y el control político ejercido sobre la Comisión en forma de preguntas parlamentarias orales o escritas.

En la actualidad, y aunque aún sea pronto para evaluar su impacto, el papel que el Parlamento Europeo está llamado a desempenar en este campo puede ser clave, ya que el procedimiento de codecisión - en virtud del cual esta institución tiene la capacidad efectiva de imponer sus enmiendas- ha sido extendido a todos los sectores de la política ambiental (con excepción de los ámbitos para los que aún se mantiene la unanimidad y de los acuerdos internacionales) por medio de las reformas del Tratado de Amsterdan que entraron en vigor en mayo de 1999.

\subsection{El Consejo de Ministros}

Por más que el Parlamento vaya adquiriendo con el transcurso del tiempo un papel más influyente en la adopción de las políticas ambientales, hoy por hoy, el Consejo sigue siendo, de lejos, la institución con más peso en el proceso decisorio.

No obstante, existen opiniones que defienden que no es en las reuniones del Consejo donde se decide, sino en los gripos de trabajo del Comité de Representantes Permanentes (Coreper) durante la negociación de las propuestas de la Comisión, enfatizando de este modo que el contenido de las decisiones que se adoptan en la Comunidad queda ordinariamente configurado en este debate entre los expertos nacionales ${ }^{12}$. Esta creencia suele emplearse como argumento para proyectar las más acerbas críticas sobre el sistema decisorio comunitario en términos de democracia, tachándosele de opaco y capturado por las élites burocráticas y censurándole el que las decisiones las acaben tomando de facto los funcionarios nacionales reunidos en los grupos de trabajo. En efecto, no puede ignorarse la influencia que el trabajo de estos grupos ejerce en la redacción de los textos finalmente adoptados y, sin embargo, también es cierto que no se dispone de investigaciones apropiadas que nos puedan confirmar hasta qué punto es cierta esa hipótesis. La confidencialidad y el secretismo que rodea los trabajos del Consejo ha impedido tradicionalmente la conformación sistemática de un substrato de evidencia empirica que confirme dichas tesis y con el que se pueda superar lo que es más una impresión o convencimiento generalizado para llegar a posibilitar una verdadera medición del peso de la actividad de estos grupos en el proceso decisorio: por políticas, por temas, por periodos, etc.

En este sentido, el trabajo de ANDERSEN y RASMUSSEN ${ }^{13}$ ha puesto de manifiesto que en el terreno de las decisiones relativas al medio ambiente la crítica referida es fruto más bien de una asunción generalizada, sin evidencia empírica. A través de su análisis, llegan a la conclusión de que la secuencia del proceso decisorio - grupo de trabajo, Coreper, Consejo- se repite varias veces para el mismo asunto antes de una decisión final a petición del Consejo de Ministros que examina los asuntos que se le someten y, ante la falta de acuerdo, reenvía los proyectos a la instancia anterior para su modificación. Se repite con cierta frecuencia el fenómeno de que los asuntos que finalmente son aprobados como punto A del orden del día del Consejo (sin debate), han sido ya objeto de discusión previamente en esta institución y reenviados a los grupos de trabajo a fin de que se reelaboraran las propuestas, de modo que, cuando vuelven a él, lo hacen con un contenido diseñado bajo el escrutinio directo de los más altos responsables políticos nacionales.

Según esta investigación, los titulares nacionales de los Departamentos de Medio Ambiente mantienen en los últimos tiempos un estrecho control sobre la actuación de los grupos de trabajo y un gran conocimiento de los asuntos especialmente -sensibles", por lo que las tesis de WeSSELS de que "en una estimación aproximada, el 80 por ciento de los actos del Consejo se deciden al nivel burocrático ", no serían ciertas para nuestra materia.

\subsection{Actores privados: participación institucionalizada y actividad de presión}

En la participación de los actores no públicos en el proceso de formulación de las decisiones comunitarias, hay que distinguir entre los foros institucionalizados y el diálogo no institucionalizado. El primero está diseñado por la autoridad pública y se crea con la intención de constituir un foro plural para la consulta de las propuestas que se estén elaborando. En el ámbito de la política ambiental comunitaria este tipo de institucionali- 
zación. se ha llevado a cabo en dos etapas, inicialmente a través de la consulta obligatoria al Comité Económico y Social integrado por los miembros que representan los intereses -socio-económicos" en la Comunidad (empresarios, asociaciones de consumidores, sindicatos, etc.) al que, por disposición del Tratado, debía formalmente sometérsele la propuesta de la Comisión una vez ésta estuviera elaborada y se hubiese transmitido al Consejo para su decisión. En fechas recientes, se ha creado un órgano ad boc, y con un sentido distinto al ECOSOC, al que se someten consultas sobre los proyectos referidos a acciones ambientales durante toda la fase de preparación de la propuesta. Se trata del inicialmente denominado aForo Consultivo General del Medio Ambiente, creado por Decisión 93/701 de la Comisión, que en 1997 pasó a denominarse -Foro Consultivo en materia de medio ambiente y desarrollo sostenible. ${ }^{15}$ compuesto por un número equilibrado de representantes de los distintos sectores interesados (industrias, asociaciones conservacionistas, sindicatos, autoridades regionales y locales y asociaciones de consumidores) ${ }^{16}$ y con respecto del cual se establece que la Comisión podrá consultarle sobre cualquier cuestión relacionada con el medio ambiente y el desarrollo sostenible.

El diálogo no institucionalizado, por su parte, se refiere a la intermediación de intereses llevada a cabo entre las autoridades públicas y los intereses afectados por la propuesta concreta de que se trate, que deciden hacerse presentes en su proceso de formulación con la intención de influir en su diseño.

En cuanto a la actuación del lobby de la industria en Bruselas, éste se realiza principalmente sobre la Comisión, si bien, con motivo de la participación cada día más intensa del Parlamento Europeo en la adopción de decisiones, nos encontramos últimamente con una alta presencia de grupos de presión en torno a los europarlamentarios. La actividad de presión puede estar basada o, no en una estructura permanente en torno a las instituciones comunitarias. En este sentido, existe un gran desequilibrio entre la participación de las grandes empresas y de las pequeñas y medianas. Estas últimas no suelen estar presentes en el proceso de toma de decisiones pues su debilidad les impide hacer valer sus intereses como grupo de presión en la esfera comunitaria ${ }^{17}$, to cual no constituye una situación diferente a la que se plantea en las arenas nacionales en las que, con la excepción de Italia en donde las asociaciones municipales de empresarios tienen cierta visibilidad pública, en el resto de los Estados niembros la ausencia de este tipo de empresas del proceso decisorio suele ser la regla. Su influencia en la formulación de medidas se debe más bien al dato de su existencia y al hecho de que el endurecimiento de las normas ambientales les afectará y, probablemente, las situará en una situación de desventaja competitiva.

En el caso de las grandes empresas la situación varía, siendo su presencia un elemento común y clave en la conformación de las decisiones. La federación que agrupa a la industria del automóvil es, por ejemplo, el principal actor en la formulación de medidas sobre los requisitos de los vehículos y sobre la exigencia de convertidores catalíticos ${ }^{18}$. Igualmente, la industria de producción de detergentes y la de recolección de envases tuvieron una alta implicación en la conformación de la Directiva sobre envases y residuos de envases, mayor incluso que los propios fabricantes de envases ${ }^{19}$. En el caso de las medidas sobre limitación de los pesticidas, los pequeños productores de estas sustancias accedieron al proceso de formulación de la propuesta de la Comisión mucho después que las multinacionales de la industria agro-química ${ }^{21}$. Todas estas empresas son conscientes de que gran parte de las decisiones que les afectan se adoptan ahora en el nivel comunitario, por lo que ante ellas se constituye un nuevo espacio de representación de intereses ${ }^{21}$.

Aparece - para las empresas que tienen los recursos financieros y humanos suficientes - un nuevo escenario de representación de intereses que se refleja en el gran aumento del número de grupos presentes en Bruselas que buscan influir eficazmente en las decisiones que les afectan. Esta situación tiene una primera consecuencia importante de cara a la articulación de la presencia de los diferentes actores en torno a la formulación de la decisión, ya que la forma en que se organizan los intereses en el nivel comunitario se corresponde más con la lógica pluralista que con la lógica corporativista ${ }^{22}$. No obstante, la apertura del sistema y su característica de no sometimiento a reglas estables hacen que los modos de representación de intereses en la esfera comunitaria no estén establecidos en forma vertical ni con interlocutores determinados de antemano, sino que es preciso emplear más bien el concepto de .red. al referirse a la presencia o no de uno o varios grupos empresariales en la conformación de una determinada política.

En cuanto a la articulación de la ‘dinámica social. en la formulación de la política ambiental, la presión en los centros de decisión comunitarios se plasma principalmente a través de las asociaciones conservacionistas ${ }^{23}$. Comparados con otros grupos de interés, estas ONG son débiles, pues suelen carecer de una mínima estructura organizativa y de unos elementales apoyos sociales que les permitan jugar un cierto papel de influencia en la arena política. Tan sólo el Worldwide Fund for Nature (WWF) y el European Environmental Bureau (que agrupa a varias asociaciones ecologistas de menor tamaño) están presentes con estructura formal estable en el lugar sede de las instituciones europeas. No obstante, la Comisión cuenta además con un registro informal de grupos de interés en el que, para el ámbito del medio ambiente, figuran otras asociaciones, lo que indica la práctica real de esta institución a incorporarles en la fase de formulación de las propuestas ${ }^{24}$.

Pese a todo, en comparación con la presencia e influencia que representa el grupo de la industria, este sector se caracteri- 
za por tener una débil representación en los procesos, en parte debido al problema estructural apuntado sobre la falta de recursos financieros y humanos que les permitan ostentar un papel más activo y debido también a que la mayor parte de estos grupos está volcada sobre la protección del medio natural, de modo que su presencia tenderá a ser mayor en las redes que se conformen en torno a las propuestas referidas a este sector específico de la política ambiental. En concreto, en la Directiva "Hábitats" (Directiva 92/43) que prevé la conformación de una Red Natura 2000 de espacios naturales en el territorio comunitario, estos grupos han participado en el proceso de formulación de la decisión y lo siguen haciendo activamente en la fase de conformación de las listas de áreas, en la que se sigue construyendo la política, si bien hay que apuntar que el grado de participación varía según los Estados y las áreas geográficas, siendo las ONG de los países del norte de Europa mucho más activas que las de los países del sur, pese a que en estos últimos la extensión de las áreas sea mucho mayor.

\section{La adopción de decisiones: el estilo decisorio de la política ambiental}

Antes de que una medida sea adoptada, una propuesta de la Comisión habrá sido:

1. Discutida en un grupo de trabajo compuesto en su mayor parte por funcionarios nacionales.

2. Sometida a un comité asesor compuesto por expertos nacionales.

3. Transmitida al Coreper para su discusión en el grupo de trabajo de funcionarios nacionales.

4. Revisada por el Coreper de nuevo.

5. Presentada al Consejo para su aprobación.

6. Desarrollada, en su caso, por la Comisión con el control de la práctica de la comitología.

A la vista de estas circunstancias, gran parte de los estudiosos de la Comunidad mantienen que es improbable que se produzca una innovación política ${ }^{25}$. Como náximo - se afirma-, la Comisión puede aspirar a generalizar y difundir soluciones adoptadas en uno o más Estados miembros introduciéndolas en la Comunidad. Las soluciones de los Estados normalmente establecen el marco para las soluciones de la Comunidad. En el caso de las decisiones en medio ambiente la innovación sería -siempre según estas posiciones- no sólo improbable sino imposible, ya que la Comunidad sólo podría actuar como una suerte de secretaría Internacional. concebida para facilitar la negociación entre los Estados miembros sobre la mejor forma de armonizar las normas que uno o varios tienen vigentes en sus territorios para alcanzar un compromiso admitido por todos, que mejorara en algo la situación de partida pero sin alejarse sustancialmente de ella. Ello nos llevaría a la consideración del incrementalismo, como modelo de toma de decisiones ${ }^{26}$.

En nuestra opinión, a pesar del pesimismo de estos juicios, la aparición de políticas innovadoras en el entorno europeo no es del todo imposible y a facilitarlo contribuye el cambio que en él se experimenta en relación a las formas de representación de intereses, desde los modelos corporativistas predominantes en las arenas nacionales, hacia unos modos de representación más abiertos y en los que existe mayor competencia entre los actores.

Esta situación tiene varias consecuencias, entre otras, la de que la forma en que se organizan los intereses en el nivel de la Comunidad se corresponde más con la lógica pluralista que con la lógica corporativista. Las relaciones que se estableceri especialmente con la Comisión a través de los "comités" muestran esta tendencia: son estructuras más abiertas que en el nivel nacional. Más específicamente, el proceso de toma de decisiones en materia ambiental se caracteriza por los siguientes rasgos que son propios de este sistema político:

- Incertidumbre. debido a la ausencia de formas estables de liderazgo. Según los sectores y según las épocas, la configuración de un sistema de actores puede variar y reestructurarse en función de las alianzas y la articulación de intereses. Debido al particular equilibrio entre los tres actores públicos principales (Comisión, Consejo asistido del Coreper y Parlamento) y a los nuevos modos de cooperación entre ellos para la toma de decisiones, no se puede determinar de modo general cuál es el elemento clave de la decisión, lo podrá ser tanto la influencia de uno de los miembros de la Comisión, o del Presidente del Parlamento Europeo o de uno de los Estados miembros o de una coalición de varios Estados. Complejidad e incertidumbre son los rasgos de la toma de decisiones que definen un panorama que hace que algunos autores ${ }^{27}$ incluyan las decisiones comunitarias en la categoría de decisiones de las que MARCH y OLSEN definieron como el modelo "papelera" (garbage can). Apoyando esta tesis estaría el fenómeno descrito anteriormente de que las acciones de política ambiental lograron la necesaria unanimidad para su adopción por parte de todos los miembros del Consejo, en gran medida debido a la inconsciencia" sobre sus repercusiones económicas y a las grandes inversiones públicas que requerian gran parte de ellas.

- Apertura: en el sentido de que el acceso a los canales a través de los que se influye en la toma de decisiones es más fácil que en el nivel nacional ${ }^{28}$. En este sentido, mientras que 
normalmente en las Administraciones nacionales nos encontramos con esquemas pertenecientes al neocorporativismo, caracterizado por la negociación estructurada entre el Estado y determinados representantes del sector afectado que monopolizan la relación, en la Comisión, sin embargo, los canales de diálogo son abiertos y, en la gestación de sus propuestas, esta institución busca el expertise multiplicando los contactos, formales e informales, con todas las partes que gravitan alrededor del tema que está siendo tratado.

- Opacidad: presente en la medida en que, paradójicamente, la apertura que se apuntaba anteriormente coexiste con un especial dificultad en comprender las reglas de juego, que lo convierten en inaccesible para los que carecen de un conocimiento profundo de las normas sobre las que se asienta el sistema y, además, no disponen de la información puntual sobre el rumbo de los trabajos de las instituciones en cada momento preciso ${ }^{20}$. El hecho de que las reglas de funcionamiento sean variables y en ocasiones ambiguas (lo que hace más abierto el sistema) contribuye a dicha opacidad.

Este complejo proceso de toma de decisiones que es, paradójicamente, a la vez abierto y opaco, termina de complicarse si además se tiene en cuenta que constantemente se debe cuestionar por métodos nada objetivos ${ }^{3 *}$ la idoneidad y la legitimidad de la acción comunitaria.

Finalmente, se observa cómo en torno a ese proceso se sitúan, de manera formal o informal, una pléyade de actores que tienen acceso a la decisión que conforman una suerte de ured. más o menos estable en cada materia manteniendo relaciones de conflicto o de alianza. Este conjunto de redes ha terminado por construir sus propios modos de acción y de relación, su propio lenguaje, etc., creando un sistema de facto inaccesible para los que están ajenos a él. Aquí se presenta la paradoja descrita por MuLre ${ }^{31}$, según la cual, al ser las reglas imprecisas, el sistema es más abierto y más fácil para ser penetrado que los sistemas burócrata-corponatistas nacionales, pero, al mismo tiempo, esas mismas reglas no están formalizadas, por lo que todas las formas de captura por determinados intereses son factibles corriéndose el riesgo de, de facto, caer en las mismas estructuras corporatistas que definen el estilo de las políticas nacionales.

Finalmente, no hay que desconocer la existencia de ciertos rasgos del proceso de toma de decisiones que se explican por las teorías neoinstitucionalistas ${ }^{32}$, en el sentido de que son las instituciones y las reglas de su actuación las que deteminan en gran medida el producto de las decisiones ${ }^{33}$. En definitiva, la forma en que está definido el proceso de toma de decisiones es crucial en la determinación de la adopción final de las normas. Los rasgos detectados que contribuirían a apoyar esta impresión son:

- la capacidad de influir en la decisión por parte de la Comisión,
- la influencia de los grupos de trabajo del Coreper en las decisiones finales del Consejo,

- el mayor peso político de los Ministros de Medio Ambiente en la arena comunitaria,

- la concepción de partida en el seno del Consejo de que es posible el consenso por más que existan diferencias iniciales en las posiciones nacionales.

\section{La implementación de las decisiones: el déficit de implementación consustancial a la política ambiental comunitaria}

Desde el enfoque de las Políticas Públicas, la fase de aplicación o de implementación de las medidas tiene una importancia extrema en la consideración global del análisis. En esta fase de la vida de una política siguen produciéndose hechos que pueden alterar, desviar o malograr los objetivos inicialmente previstos por la medida. No puede sostenerse realmente que exista una clara y neta separación entre los actores que participan en la formulación de una política y los órganos y sujetos que han de ponerla en práctica. En definitiva, es preciso hacer hincapié en la fase de producción de resultados, porque los desajustes o déficits que se produzcan en esta etapa están directamente conectados a las características o circunstancias del proceso de la adopción de las medidas y porque su exploración ofrece los elementos que permiten ir corrigiendo las posibles deficiencias, irregularidades o anomalias, de modo que cada acción pueda convertirse en un sistema que se retroalimenta sobre los efectos de su propia evaluación.

En la legislación ambiental comunitaria, precisamente nos encontramos ante un caso en el de déficit de implementación ${ }^{16}$ de los más agudos y serios que enfrenta la Comunidad con respecto a sus diferentes políicas. Los informes que anualmente prepara la Comisión sobre la aplicación de las medidas ambientales muestran un panorama muy negativo, caracterizado por un alto índice de falta de aplicación real de las directivas comunitarias. El número de expedientes abiertos por la Comisión contra los Estados por incumplimiento de las medidas ambientales y de recursos planteados ante el Tribunal de Justicia también es el más alto de todos los ámbitos de actividad comunitaria. 
En términos generales y abstractos se considera de general asunción que los avatares que puedan presentarse en la ejecución de un programa están en función de tres factores principales: las características del programa, los comportamientos de los ejecutores reconocidos y las reacciones de los grupos-objetivo. En el caso de la Política Ambiental de la Comunidad, estos tres factores se conjugan para propiciar una insatisfactoria aplicación de las medidas. El proceso de implementación de la política ambiental comunitaria envuelve a diferentes niveles gubernamentales y órganos administrativos que, en muchos casos, no tienen la coordinación adecuada para propiciar una acción conjunta en la aplicación.

Adicionalmente, en ocasiones los programas no están definidos de forma clara, o bien su enunciación permite una interpretación que desvía la ejecución de los objetivos primigenios de la política. La redacción de las Directivas en ocasiones no tiene debidamente en cuenta la diversidad de situaciones y las condiciones del medio ambiente en las distintas regiones de la Comunidad o bien no se toman en consideración los distintos ordenamientos y prácticas jurídicas y administrativas de los Estados miembros.

Al tratarse de Directivas, los problemas de aplicación suelen ser de dos órdenes: de incorporación al ordenamiento interno o de aplicación práctica. El primero está prácticamente resuelto, con algunas excepciones que aparecen anualmente en los informes de aplicación del Derecho comunitario y que están relacionados con las prescripciones constitucionales de algunos Estados, relativas a las estructuras territoriales de reparto del poder (es el caso típico de Bélgica) o con la práctica de algunos Estados de transponer directivas por medio de circulares (como en Alemania ${ }^{35}$ ). En otras ocasiones, las dificultades se derivan de las incidencias de las disposiciones ambientales de otros ámbitos de acción del Estado (agricultura, transportes, industria) 0 en los casos en que los Estados disponen de una legislación previa nacional que cubre el sector regulado por la Directiva y que debe modificarse para garantizar que se ajusta a los nuevos requisitos comunitarios.

Sin embargo, en los momentos actuales, los problemas son de aplicación práctica y de control de cumplimiento ${ }^{37}$, entendiendo por •aplicación práctica la incorporación de normas comunitarias por parte de las autoridades competentes en decisiones concretas y por "control del cumplimiento" todas las intervenciones de las autoridades competentes para incitar $u$ obligar a otros a respetar lo estipulado (control, inspecciones puntuales, sanciones, medidas correctivas vinculantes), con objeto de mejorar los resultados de la política de medio ambiente.

El balance sobre la ejecución de la Política Ambiental europea arroja, como hemos puesto anteriormente de manifiesto, unas cifras desoladoras ${ }^{38}$ :
- en 1993 la Comisión registró un pico en la tendencia en este ámbito, con un total de 383 denuncias sobre supuestas infracciones, entre denuncias, preguntas parlamentarias y casos detectados por la Comisión, que equivale al 28,5\% de todos los casos. En 1994, la cifra se rebajó a 359 (25\%), en 1995: 265 casos (20\%), en 1996 bajó a 207 (20\%). Desde 1990 hasta la fecha, España, Francia, el Reino Unido y Alemania son los Estados contra los que se presentaron mayor número de quejas ${ }^{3}$, siendo Luxemburgo y Dinamarca los menos afectados ${ }^{\text {in }}$;

- en 1997, el Tribunal de Justicia presentó 37 recursos por incumplimiento ante el Tribunal de Justicia y envió 69 dictámenes motivados;

En las Comunicaciones anuales de la Comisión al Parlamento Europeo y al Consejo se distingue entre falta de transposición de directivas y aplicación incorrecta de las mismas, detallando, por Estados y por sectores, dónde se encuentran los principales problemas. Los problemas están repartidos por todos los sectores, pero, tal vez, del examen de los últimos informes se pueda destacar que las mayores dificultades se dan en materia de aguas y en materia de protección de la naturaleza. Asimismo, se experimenta un desarrollo creciente de las dificultades en el sector de los residuos, derivado del aumento del volumen y peligrosidad de los mismos y de las medidas, cada vez más estrictas, que se han adoptado en el nivel comunitario. También se insiste en todos y cada uno de los informes, de la deficiente aplicación práctica de las Directivas 85/337 sobre evaluación de impacto y $90 / 313$ sobre libre acceso a la información, sectores en los que se recibe de forma estable un gran número de quejas ${ }^{41}$.

En España, los mayores problemas en cuanto a la aplicación incorrecta se encuentran, de forma inalterable por el transcurso del tiempo, principalmente en las cuestiones sobre protección de la naturaleza, la incorrecta aplicación de la Directiva sobre evaluación de impacto ambiental, el agua y los residuos.

De entre las causas por las que en este ámbito se produce este fenómeno tan acusado de déficit de implementación, se podrían señalar las siguientes:

1. La complejidad técnica y jurídica de la materia, que provoca muchos problemas de interpretación y aplicación técnica y plantea dificultades de coordinación entre las distintas autoridades nacionales implicadas en las fases de incorporación al ordenamiento interno, aplicación práctica y cumplimiento.

2. Dentro de los Estados miembros, tanto federales como unitarios, la competencia en relación con las responsabilidades públicas en la aplicación práctica y el control del cumplimiento suele estar compartida o atribuida a distintas esferas de la Administración. Esta descentralización añade compleji- 
dad y, por tanto, exige el establecimiento de una coordinación en el interior de lo que la Comisión llama la "cadena reglamentaria " ${ }^{12}$ para conseguir una ejecución correcta y plena.

3. La complejidad de las funciones de inspección y control requiere personal suficiente, convenientemente cualificado y con los recursos adecuados, situación que no se da siempre en todos los Estados miembros. Existen grandes disparidades entre los organismos y los mecanismos coercitivos de los Estados miembros, y mientras algunos han asignado importantes recursos a los cuerpos de inspectores y demás organismos que supervisan la aplicación práctica de las políticas comunitarias, otros han tomado menos medidas o no han adoptado ninguna en absoluto ${ }^{\text {i3. }}$.

4. El procedimiento del artículo 169 del Tratado, por el que la Comisión, como guardiana de los Tratados, está llamada a perseguir los incumplimientos de los Estados, no es suficiente como instrumento de control. Es un procedimiento largo, pesado y en exceso formalista. Es imposible que con un único sistema comunitario judicial para hacer cumplir la legislación, puedan abarcarse todos las estructuras administrativas locales, regionales, privadas, etc., a través de las que se ejecuta —en aplicación del principio de administración indirecta - las políticas ambientales comunitarias. Además, la mayoría de las quejas denuncian problemas específicos, muy concretos, que afectan directamente a los demandantes (ej. en los casos de las quejas relativas a la Directiva 85/337 sobre EIA o a la Directiva 79/409 sobre aves). En definitiva, se necesitan otros medios más eficaces que puedan dar pleno efecto a las normas europeas.

5. La Comisión sólo dispone de poderes y recursos limitados, está en una situación de dependencia casi total con respecto a la información que se le comunica con carácter ad boc a través de denuncias, peticiones, preguntas orales y escritas al Parlamento, $\mathrm{ONG}^{14}$, y medios de comunicación. Aunque esta información tiene mucho valor, la imposibilidad de verificación directa puede tener consecuencias negativas para el medio ambiente y para el prestigio del sistema, en el caso de que las denuncias sean infundadas.

6. Los problemas inherentes a la activación de la acción judicial. Es preciso reconocer de antemano que la aplicación del Derecho por medio del recurso a la justicia está garantizado de facto cuando hay intereses económicos en juego y a las partes les resulte "rentable. litigar. Un sistema para hacer cumplir normas encaminadas a constituir un marco para una actividad económica, puede obtener el apoyo de los sectores industriales, agrarios, comerciales, etc., con recursos económicos suficientes para luchar por su aplicación ${ }^{\text {} 5}$. En el caso de los intereses "ecológicos" esto no ocurre, pues no hay ningún sector económicamente relevante (y con recursos disponibles para entablar litigios) que pueda desempenar el papel de supervisores privados del cumplimiento de las normas de medio ambiente y se alcen como sus defensores. Las ONG no suelen disponer de recursos para litigar judicialmente, carecen tanto de recursos económicos como humanos y de información. Por ello, se depende de las autoridades públicas y de sus potestades, recursos y buena voluntad para posibilitar una correcta implementación de las politicas ambientales. Pero ello lleva a resultados insatisfactorios, teniendo en cuenta que muchas veces se produce el efecto de poner al lobo a cuidar a las gallinas", pues la Administración es la que de modo directo (porque se trata de explotaciones públicas en algunos casos) o indirecto (porque está en connivencia con los agentes económicos que producen la contaminación y que tienen a las autoridades sometidas al chantaje del cierre de la instalación) es la principal responsable de la falta de cumplimiento.

La política ambiental comunitaria únicamente será eficaz si los Estados miembros aplican y hacen cumplir plenamente las medidas acordadas en el nivel europeo, puesto que, en la medida en que son ellos los agentes implementadores de las políticas, la solución deberá partir de su voluntad ${ }^{16}$.

La protección del medio ambiente implica retos específicos que la hacen en muchas formas distinta de otros temas de política y legislación y que esto debería tomarse en consideración al redactar, aplicar y hacer cumplir el Derecho en esta materia. La Red europea para la aplicación y el cumplimiento de la legislación en materia de medio ambiente (IMPEL), por ejemplo, creada por previsión del Quinto Programa de Acción, ha desempeñado un papel útil como red informal para detectar los problemas en la aplicación, la inspección y el cumplimiento de las Directivas ambientales comunitarias ${ }^{47}$, en concreto en los temas relacionados con la contaminación industrial.

\section{La nueva estrategia de la política ambiental europea}

En torno a 1993 se produjo un revulsivo en los términos en que pasa a ser concebida la política ambiental comunitaria. Su causa tiene en parte que ver con los efectos de la crisis de Maastricht, pero también tiene influencia en el cambio hacia un 
nuevo enfoque otro hecho fundamental que coincide en el tiempo: la adopción del Quinto Programa de Acción a comienzos de 1993, en el que se diseña una nueva estrategia comunitaria para el tratamiento de los problemas ambientales.

No obstante, el momento de inflexión de la política ambiental en el que nos encontramos, a diferencia de lo que pudiera parecer, no se produce exclusivamente por el efecto concatenado de los dos fenómenos referidos. En nuestra opinión, la voluntad de lanzar una nueva estrategia se debe a un efecto combinado entre varios factores:

1. Por la reforma de Maastricht. En este caso, no se trata tanto de las modificaciones concretas operadas en el Tratado que pudieran afectar a nuestra política, pues la aplicación del principio de subsidiariedad ya se venía realizando en este campo, por ser una de las condiciones bajo las que había surgido esta política en tiempos del Acta Única Europea. El verdadero detonante para el cambio es la crisis post-Maastricht y los efectos que produce de actuar con mucha más cautela en el desarrollo de la acción comunitaria, debido a los recelos expresados por algunos Estados miembros con respecto a la excesiva expansión de la competencia de la Comunidad hacia la mayoría de los ámbitos de actuación pública. En el terreno del medio ambiente, las críticas al excesivo detalle con que se acometía el tratamiento público del sector redundan en un cambio hacia fórmulas más suaves de las que las Directivas marco, como la referida a la calidad del aire ambiente o la de control integrado de la contaminación son ejemplos significativos.

2. El sonado fracaso de la implementación de las políticas ambientales. Como acabamos de referir, el panorama sobre el efectivo cumplimiento de las Directivas ambientales es francamente negativo y los informes que anualmente prepara la Comisión denuncian el deficiente rendimiento de las normas en prácticamente todos los sectores del medio ambiente, en especial con respecto de los instrumentos que aplica técnicas de objetivos de calidad o aquellos que se sirven de instrumentos procedimentales, en los que los Estados suelen tender a aplicarlos de forma muy flexible que, en ocasiones, lleva a un alejamiento de los objetivos que perseguía la norma europea. La nueva estrategia, por tanto, diseña algunos instrumentos que podrían contribuir a paliar el déficit de implementación que afecta al sector. En este sentido, la conformación de la red IMPEL para el estudio de los problemas de aplicación de las normas ambientales, la puesta en marcha de la Agencia Europea de Medio Ambiente encargada de recabar información que permita conocer el estado del medio ambiente y, con ello, disenar mejores políticas, o los esfuerzos por incorporar a todos los grupos de interés en el desarrollo de las acciones comunitarias (a tavés de financiación de actividades de las ONG por ejemplo) son signos que indican, en nuestra opinión, una vía para el cambio.

3. El cambio de enfoque del Quinto Programa de Acción que adopta una visión más amplia sobre los instrumentos que se deben aplicar. Aquí nos detendremos un poco más debido a la importancia de los nuevos conceptos que incorpora este documento:

La nueva estrategia consiste en centrarse menos en la adopción de medidas sectoriales sobre acciones especificas y más en el intento de producir una modificación de las tendencias actuales de comportamiento que contribuyen al deterioro del medio ambiente y al agotamiento de los recursos naturales. Asimismo, está basado en el principio de subsidiariedad y responsabilidad compartida. Por tanto, en el establecimiento de las prioridades que efectúa, la selección está basada más en indicar grandes orientaciones que en el diseño de acciones concretas y, así, dispone la realización de medidas horizontales a fin de cambiar los hábitos de consumo y el comportamiento de la sociedad para lograr una mayor compatibilidad con el concepto de desarrollo sostenible. En este nuevo enfoque, aunque el planteamiento a través de políticas regulativas sectoriales seguirá cumpliendo una función importante, es preciso añadir unas medidas complementarias, dándose prioridad a las siguientes: mejora de los datos (información básica, tendencias, indicadores), fijación correcta de los precios (internalización de los costes externos mediante mecanismos de valoración y determinación del coste, análisis coste/beneficio, incentivos económicos y fiscales valoraciones ecológicas, responsabilidad civil, etc.), información, educación y formación de todos los actores económicos (incluidos los políticos, planificadores, gestores, trabajadores y consumidores), plena integración del medio ambiente en las demás políticas y una preocupación mayor por una aplicación y vigilancia estrictas.

Asimismo, al respetar el principio de subsidiariedad y el concepto de responsabilidad compartida, muchas de las acciones arriba mencionadas se llevarán a cabo desde distintos niveles de gobierno. El nuevo enfoque combina el concepto de los nuevos instrumentos de política ambiental con el concepto de corresponsabilidad de todos los actores en el diseño y en la implementación de las medidas. En efecto, se trasciende la filosofía de la subsidiariedad para acuñar el concepto más amplio de responsabilidad compartida, término que implica, no tanto la selección de un determinado nivel de gobierno en detrimento de otro, sino más bien una intervención mixta de actores e instrumentos en los niveles adecuados, sin poner en cuestión la división de competencias entre la Comunidad, los Estados miembros, las autoridades regionales y locales, los agentes económicos y los sociales. Igualmente se puede observar el espa- 
cio limitado que tienen las políticas regulativas en este nuevo enfoque, mientras que el resto de medidas: acuerdos voluntarios, instrumentos de mercado, información, $I+D$, etc., se integran de forma coherente con el resto de las medidas.

Hasta la fecha las nuevas decisiones que están siendo adoptadas recogen en gran medida este enfoque. Hay ejemplos variados sobre la recepción de este nuevo enloque en la política ambiental elaborada en los últimos años, como las políticas que establecen sistemas europeos de ecoetiquetado y ecoauditoría, o la Directiva 96/61 sobre el control integrado de la contaminación. Esta última, en concreto, que podría considerarse como el -buque insignia. del nuevo enfoque, considera que el tratamiento por separado de las emisiones a la atmósfera, el agua o el suelo, puede potenciar la transferencia de contaminación entre los diferentes ámbitos del medio ambiente, en lugar de proteger al medio ambiente en su conjunto y trata de acabar con las llamadas end of pipe solutions, las cuales, al tratar aisladamente la contaminación en un medio determinado (aire, agua), provocan una traslación de la contaminación de un medio a otro. Esta -tercera generación" de iniciativas comunitarias en materia de medio ambiente ${ }^{\text {ts }}$ favorecen un enfoque global, respetan el principio de subsidiariedad y promueven la participación de toclos los actores interesados y de la sinergia entre la industria y el medio ambiente.
En definitiva, en la actualidad estamos ante un momento en el que los seis años transcurridos desde los últimos detonantes del cambio (Quinto Programa y crisis post-Maastricht) permiten ver si los modos en que en la actualidad se están conformando las decisiones comunitarias suponen o no un cambio con respecto a la dinámica anterior. Creemos que la respuesta es positiva y que en efecto nos encontramos ante un nueva etapa de la construcción de la política ambiental que no es tributaria del rumbo actual de la evolución de la integración europea, sino de las características singulares y propias del problema que esta acción comunitaria enfrenta. El deterioro del medio ambiente, que hoy por hoy mantiene una tendencia al agravamiento "i), sigue demandando políticas activas por parte de todos los niveles de gobierno, entre otros del gobierno europeo, por lo que la política ambiental europea intensifica su labor y se extiende hacia otros ámbitos. Por otro lado, los problemas de aplicación práctica detectados en el transcurso de la vida de esta política permiten retroalimentar el sistema e intentar proceder a un ajuste de elementos alli donde se detectan los fallos. Finalmente, se produce un cambio en cuanto a las técnicas políticas que pasan a trascender de las políticas meramente regulativas para extenderse hacia otros cambios (instrumentos de mercado, políticas redsitributivas, información, educación, etc.), marcando igualmente un cambio en el paso de esta acción comunitaria hacia el nuevo siglo.
- Profesom del Departamento de Ciencia Política y de la Adiministración de la Universidad Aulónoma de Madrid.

1 Piénsese por cjemplo que, en este final de siglo, el volumen de recursos que mueven el conjunto de las Admunistraciones Públicas supone la mitad de la riqueza que un páis produce cada año. En España el presupuesto püblico está en torno al $46 \%$ del PIB, siendo la media europea el $50 \%$.

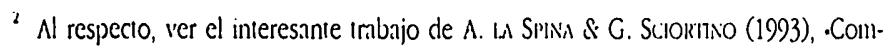
mon agenda, southern rules: European Integration and Environmental Polic;:, en I.D.

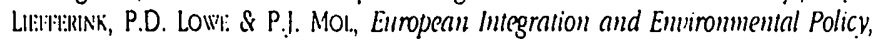
Bellhaven Press, London-New York, págs. 217-237, en el que se explora, entre otras materias, la concreta percepción sobre los problemas ambientales de las sociedades litlinas y su correspondencia con la participación en la formulación de las políticas.

A. Siskicin (1996), Environmental policy: the pusht-pull. of policy:-making, en $\mathrm{H}$.

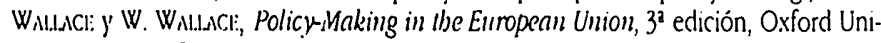
versity' Press, Oxford. pp. 235-255.

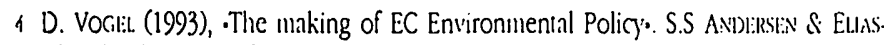
slis (ceds.), The Europeification of National Policy-making. London, p. 19.

${ }^{3}$ A. Mol. y D. Lli:iti:kINk (1993), European environmental policy and global interde-

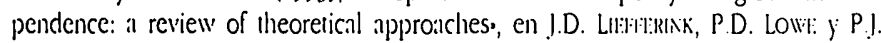
MOl., op. cil., European Integration and Environmental Policy, pp. 17-35.

c Vid. L. Krïst:R (1992), Focus on European Enmirommental, Sreet \& Maxwell, London; (1998) E.C. Treaty and Enmirommental Law. $3^{2}$ edición. Sweet \& Maxwell, London.

' En cuya categoria ahom habria que añadir a los nuevos países de la uiltima adhesión: Austria, Finlandia y Suecia, en especial este último, que practica políticas anlbientales muy avanzadas.
3.Q. WILSON (ed.) (1980), The politics of regulation, Nen York, Basic Books.

' G. Minjon: (1996), -The rise of statutory regulation in Europe, en Mnjon:, G. (ed.) Regulating Europe, London, Routledge, pp. 48-60.

"' G. MiJON:, op. cit.

"La Comisión de Medio Ambiente, Salud Pública y' Protección del Consumidor, con 51 miembros, es una de las más numerosas y activas dentro de la actividad del Parlamento. Sin embargo, en opinión de Susana AGUILsk Fl:R:Niviv):\% (op. cit., El reto del medio ambiente: conflictos e intereses en la politica medioamabiental, Alianza, D.I., Madrid, 1997): a pesar de que en esta Comisión la predominado un alto nivel de consenso debido a que las diferencias partidistas parecen difuminarse a la hom de iratar temas ambientales, su carencia de expertise para elaborar propuestas ha limitado su potencial influencia. (p. 76).

1" Vid. WhLLACI: $:$ WALLICE: (eds.), op. cit., (Policy Making in the European Union).

"M.S. ANIDERSEN Y L.N. RASIIUSSI:N (1998), The Making of Environmental Polic; in the European Council., Joumal of Common Market Studies, vol. 36, N.. 4, p. 585-97.

14 W. Wl:si:1s (1991), The EC Council: The Communiry's Decisionmaking Center. En R.O. Kl:OIn:N: y S. HOHMin (eds.), The Nenn European Commmity: Decision-Making and Institutional Change. Westriew, Boulder.

1) DOCE L 58 de 27 de febrero de 1997.

${ }^{16}$ Más en concreto, la Decisión que lo crea establece en su articulo 3 que estará compuesto de 32 miembros distribuidos según el siguiente reparto: entre siele y doce puestos para personalidades procedentes de empresas, entre tres $y$ cinco puestos para representantes de autoridades regionales y locales, entre cuatro y siete puestos para representantes de organizaciones de defensi del medio anbiente y de los con- 
sumidores, entre uno y tres puestos para representantes de los sindicatos, entre siete ¡ diez puestos para personalidades eminentes.

${ }^{17}$ F. LEV:Qu: (1996), The European Fabric of Environmental Regulations, en Emirommental Policy in Europe. Industry, Competition and the Policy Process, Ednard Elgar, Cheltenham-Brookfield. pp. 9-30.

18 Vid. T. WIISTON \& M. GLICHANT (1996), V Voluntary Agreements berrieen Industry and Government, en Llivi:Qui: (ed.), op. cit. (Enuirommental Policy in Europe...), pp. 143-173.

19 Vid S. Brusco, P. Beiriossı \& A. Comen (1996), Playing on ino Chessboards: the European Waste Management Industry, the Strategic Behaviour in the Market and in the Policy Debate, en Livi:Qur: (ed.), op. cit. (Enmironmental Policy in Europe), pp. 113-142.

"A. NADiI (1996), from Environment to Competition. The EU Regulatony Process in Pesticide Regisimation, en Livv:oul:, op. cit., pp. 53-74.

?V Vid., entre otros, P. Mulli:k (1995), . Un espace européen des politiques publiques, en Y. ME.ry, P. Mulul: IJ. L. Qui:RMon: (coords.), Politiques Publiques en Europe, L'Harmaltan, Paris, pp. 11-24; J.L. Qul:kMoNn: (1994), Le sustène politique de l'Union Européenne, segunda edición, Monichrestien, Paris.

"Iid infra.

"Para una aproximación genemal a la materia, vid. S. BockING (1997), Ecologists and Emironmental Politics. A History of Contemporary Ecology, Yale University Press, New Haven and London.

:1 Amigos de la Naruraleza Internacional, Birdlife Internacional, Creenpeace Intemacional, Eurogroup (incluye a la Asociación Nacional de Defensa de los Animales espanola), Anligos de la Tierra, Fundación para la Política Ambiental Europea y Coalición Europea para acabar con los experimentos en los animales, entre otras.

is G. Mnon: (ed.) (1996), Regulationg Europe, London, Routledge, 1996

s C.E. LiNibiton (1959), -The Science of Muddling Through, en Pulblic Administra. tion Rerielli, vol 19.

27 P. Mullt: (1995), Un espace européen des politiques publiques, en op. cit. Y. MiNy, P. Mul.Li:R f: I.L. Qul:RuONI: (coords.), Politiques Publiques en Europe, p. 20.

.8 S.A. ANII:RSI:N y K.A. El.InSSLiN (1993), Policy-mllaking and the institutions en the EC, en Making Policy in Europe, the Europeification of National Policy-Making, Sage, London.

to Véase, J. Husurlik:YYs (1997), Negotiating in the European Union: bow to make the Brissels Macbine Work for your. Century, London.

" Como la aplicación del Principio de Subsidiariedad y el Principio de Proporcionalidad que son condicionantes básicos para la activación de la competencia de la Comunidad Europea.

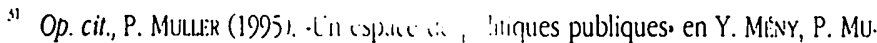
Lu:R y J.L. Qut:Ruon: (coords.), Politiques Publufu' ‘'ll Eumope, L'Harmattan, Paris.

"2 I.G. MARCH Y J.P. OLSI:N (1993), El nuevo instifucionalismo: factores organizativos de la vida políica, en Zöna Abierta 63/64.

"En este sentido, especialmente referido al funcionamiento de la Comunidad, inid. M.A. Poluck (1996), The new institutionalism an EC Govemance: the promise and limits of Institutional analysis, en Governance, vol. 9, $n^{2} 4$, pp. 429-458. y B. Kl:RR:MANs (1996), Do institutions make a difference? non-institutionalism, neo-institutionalism and the logic of Common Decision-Making in the European Union, en Goternance, vol. 9, $n^{2} 2$, pp. 217-240.

* Entendemos por déficit de implementación el fenómeno de inadecuación entre los objetivos previsios en la norma y los resuliados de facto producidos.

${ }^{5} \mathrm{Vid}$. Undécimo Informe anual sobre la aplicación del Derecho comunitario (1993) COM (04) 500 final de 29 de marzo de 1993, p. 92.
36 Comunicación de la Comisión: Aplicación del Derecho comunitario del medio ambiente. COM(O6) 500 final, de 22 de octubre de 1996.

3 Por ejemplo, a la hora de conceder una autorización o de declarar protegida una zona de especial interés nanural. Este concepto también incluje la disponibilidad de los medios y' recursos para que las autoridades competentes puedan cumplir las obligaciones que les incumben en virtud del Derecho comunitario y que puedan de facto adoptar las decisiones adecuadas.

$\$ 8$ Extraidas de los últimos informes de aplicación del Derecho comunitario: Undécimo Informe anual sobre la aplicación del Derecho comunitario (1993) COM (04) 500 final de 29 de marzo de 1993; Duodécimo Informe anual sobre la aplicación del De. recho comunitario (1994) COM (95) 500 de 7 de junio de 1995; Decimotercer Informe anual sobre la aplicación del Derecho comunitario (1995) COM(06) 600 final, de 29 de mayo de 1996; Decimocuarto Informe anual sobre la aplicación del Derecho co. munitario (1996), COM(07) 299 final de 29 de majo de 1997; Decimoquinto Informe anual sobre la aplicación del Derecho comunitario (1997), COM(98) 317 final, de 19 de mayo de 1998.

39. Lo cual no significa que en el balance final sean ellos los más sancionados, piénsese que muchas de las quejas no se sustancian posteriormente en infracciones, bien porque no existe un fundamento juridico de Derecho comunitario, bien porque las alegaciones de los demandantes o solicitantes carecen de fundamento, de hecho o de derecho.

40 Vid. Decimotercer Informe anual sobre la aplicación del Derecho comunitario (1995) $\operatorname{COM}(06) 600$ final, de 29 de mayo de 1996, p. 90.

"Las quejas se refieren en la majoria de las ocasiones a la negativa de las Administraciones nacionales a dar curso a las solicitudes de información o a la exigencia de pagar cánones cuyo importe va más allá de lo razonable. Decimocuarto Informe anual sobre la aplicación del Derecho comunitario (1996), COM(97) 299 final de 29 de majo de 1997, p. 106.

4: Este concepto hace referencia al proceso completo de determinación, concepción, proyecto, adopción, ejecución y cumplimiento de una norma legislativa hasta la evaluación de su eficacia. Se irata de una herramienta metodológica que posibilita la aplicación del enfoque diolistico a los insirumentos de la politica ambiental. Vid. Informe de la Comisión sobre la aplicación del Derecbo commitario del medio ambiente, COM(O6) 500 final, de 22 de octubre de 1996.

${ }^{43}$ Vid., op. cit., Informe de la Comision sobre la aplicación del Derecbo communitario del medio ambiente, p. 13

"La Comisión, en su XV Infonme sobre la aplicación del Derecbo comunitario (op. cit., p. 85), destaca la urilidad particular, para apreciar la aplicación efectiva de las normas comunitarias, que tienen algunas quejas muy documentadas, procedentes a menudo de organizaciones no gubernamentales.

45 Éste es uno de los mayores problemas que identifica Krisi:R como causa de la falta de aplicación príctica del Derecho ambiental comunitario, en L. KRiMl:R (1997), -Deficits in application of EEC environmental law and its causes. Perspectives de Dret Comunitari Ambiental. Bellaterra, pp. 471.495.

* R. Microry (1992), The enforcement of Community Environmental Lams: some critical issues. Common Market Lam Retien", $n^{2} 29$, pp. 347-369.

"Resolución del Consejo de 7 de ocrubre de 1997 sobre la redacción, aplicación y cumpliniento del Derecho comunitario en materia de medio ambiente. (DOCEC 321 de 22 de ocrubre de 1997).

${ }^{48}$ Así la define la Comisión en su Decimocuarto Informe anual sobre la aplicación del Derecho comunitario (1996), COM(07) 299 final de 29 de mayo de 1997.

4) Vid. Agencia Europea de Medio ambiente (1998) II Informe sobre el estado del medio ambiente en Europa, AEMA, Copenhague.

\section{Bibliografia}

ANDIRSEN, M.S. y RASMUSSSEN, L.N. (1998), The Making of Environmental Policy in the European Council, Joumal of Common Market Studies, vol. 36, n. 4 , pp. 585-97.
(1993), Policy-making and the instirutions en the EC, en Making Policy in Europe, the Europeification of National Policy-Making, Sage, London. 
Bockinc, S. (1997), Ecologists and Enmironmental Politics. A History of Contemporary Ecology, Yale University Press, New Haven and London.

Kl:RRl:MNS, B. (1996) •Do institutions make a difference? non-institutionalism, neo-institutionalism and the logic of Common Decision-Making in the European Union, en Govenuance, vol. 9, n² 2, pp. 217-240.

Krïu:k, L. (1992), Focus on European Enirommental, Sreet \& Maxtell, London.

_(1998), E.C. Treaty and Enmironmental Law: $3^{\mathrm{a}}$ edición. Sreet \& Maxwell, London.

LII:IF:RINK, J.D., Lowil, P.D. \& MOl., P.J. (1993), European Integralion and Enuironmenral Policy, Bellaven Press, London-New York, pp. 217-237.

LI:vi:Qul:, F. (1996), Enuronmental Policy in Europe. Industry, Competition and the Policy Process, Edward Elgar, Chelienlam-Brookfield.

Mis.rony, R. (1992). The enforcement of Community Environmental Lanis: some critical issues. Common Market Lam Renien, n² 29, pp. 347-369.

Miуos:, G. (ed.) (1996), Regulating Europe, London, Routledge, 1996.

M,^RCII, I.G. y OLs:iN, J.P. (1993), EEl nuevo institucionalismo: factores organizativos de In vida politica, en Zoma Abierta 63/64.

Mi:Ny, Y., MUu,Li:R, P. \& QUi:RNoNi:, I.L. (coords.) (1995), Politiques Publiques en Europe, L'Harmattan, Paris, pp. 11-24.
Pousıck, M.A. (1996), The new institutionalism an EC Governance: the promise and limits of Institutional analysisi, en Gorenance, vol. 9, $n^{2} 4$, pp. 429-458.

Qul:Rionn:, J.L. (1994), Le système politique de l'Union Européenne, segunda edición, Montclirestien, Paris.

SBR木Gis, A. (1996), Environmental policy: the push-pull, of policy-making, en $W_{A}$ LLscl, H. y Willsc:, W. Policy-Making in the European Union, $3^{a}$ edición, Oxford University Press, Oxford, 1996, pp. 235-255.

_(1993), E.C. Enviromental Policy: Aripical Ambitions and Typical Problems, en $\mathrm{C}_{1}$ IRUNy, A.W. , Roslinmint., G.G., The State of the European Communiy. Longman. Colorado.

Vocili, D. (1993), The making of EC Environmental Polic);. ANIJ):Rs:N, S.S. \& ELlissi:N (eds.), The Europeification of National Policy-making. London, p. 19.

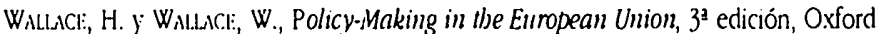
University Press, Oxford, pp. 235-255.

Wissi:Ls, W. (1991), The EC Council: The Community's Decisionmaking Center. En Krollanl:, R.O. y Hol:Mnas, S. (eds.), The Netn European Community. Decision-Making and Institutional Change. Westview, Boulder.

WIISON, J.Q. (ed.) (1980), The politics of regulation, Nen York, Basic Books. 
